\title{
Percepções Familiares Sobre a Visibilidade do Enfermeiro Atuante em Unidade Intensiva Neonatal e Pediátrica
}

\author{
Silviamar Camponogara ${ }^{1}$, Camila Pinno ${ }^{2}$, Constância Nenê Loiola ${ }^{3}$, \\ Carla Silveira de Oliveira ${ }^{4}$, Lenize Nunes Moura ${ }^{5}$, Gisele Loise Dias ${ }^{6}$
}

\begin{abstract}
RESUMO
Objetivo: conhecer a percepção dos familiares sobre a visibilidade do enfermeiro atuante em Unidade de Terapia Intensiva neonatal e pediátrica. Metodologia: trata-se de um estudo descritivo e exploratório com abordagem qualitativa. A coleta de dados ocorreu por meio de entrevista semiestruturada com 12 familiares de pacientes internados nas Unidades de Terapia Intensiva Pediátrica e Neonatal em um hospital universitário localizado no interior do Estado do RS. A análise dos dados seguiu os pressupostos da análise de conteúdo. Resultados: Após a análise das entrevistas, emergiu a seguinte categoria temática: "Visibilidade do trabalho do enfermeiro em Unidade de Terapia Intensiva neonatal e pediátrica": os participantes mencionaram suas percepções sobre a Unidade de Terapia Intensiva (UTI), bem como sobre o trabalho do enfermeiro ante as atividades de assistência e gerência, na qual mencionam o reconhecimento da atuação do enfermeiro no cuidado direto aos pacientes, assim como no desenvolvimento de ações educativas, tendo como base uma prática humanizada. Conclusão: $O$ trabalho do enfermeiro alcança visibilidade no setor investigado por meio de ações de cuidado direto e educativas por parte dos familiares. Palavras-chave: Enfermeiros. Família. Unidade de Terapia Intensiva.
\end{abstract}

FAMILY MEMBER PERCEPTIONS ON THE VISIBILITY OF THE ACTING NURSE IN THE NEONATAL AND PEDIATRIC INTENSIVE UNIT

\begin{abstract}
Objective: to get to know the perception of family members about the visibility of nurses working in neonatal and pediatric intensive care units. Methodology: This is a descriptive and exploratory study with a qualitative approach. Data was collected through a semi-structured interview with 12 relatives of patients hospitalized at the Pediatric and Neonatal Intensive Care Units, in a university hospital located in the state of RS. The analysis of the data followed the assumptions of content analysis. Results: After analyzing the interviews, the following thematic category emerged: "Visibility of the work of the nurse in a neonatal and pediatric intensive care unit": the participants mentioned their perceptions about the intensive care unit (ICU) as well as the work of the nurse in front of the activities of care and management, in which they mention the recognition of the nurses' performance in the direct care to the patients, as well as in the development of educative actions, based on a humanized practice. Conclusion: The work of the nurse reaches visibility in the researched sector, through the visualization of direct care and educational actions, by the family members.

Keywords: Nurses. Family. Intensive care unit.
\end{abstract}

Recebido em: 6/4/2018

Aceito em: 20/9/2018

\footnotetext{
${ }^{1}$ Enfermeira. Doutora em Enfermagem. Docente no curso de enfermagem e PPGEnf/UFSM. Universidade Federal de Santa Maria. silviaufsm@yahoo.com.br 2 Enfermeira. Doutoranda do Programa de Pós-Graduação em Enfermagem da Universidade Federal de Santa Maria (PPGENF/UFSM). pinnocamila@gmail.com

${ }^{3}$ Enfermeira. Especialização em Enfermagem em Suporte Avançado de Vida-Urgência e Emergência. Ufam. consloiola15@hotmail.com

${ }^{4}$ Enfermeira. Graduada pela Universidade Federal de Santa Maria Graduada - UFSM. carlasoenf@gmail.com

${ }^{5}$ Enfermeira. Mestranda do Programa de Pós-Graduação em Enfermagem da Universidade Federal de Santa Maria (PPGENF/UFSM). lenize.nunes@hotmail.com ${ }^{6}$ Enfermeira. Doutoranda do Programa de Pós-Graduação em Enfermagem da Universidade Federal de Santa Maria (PPGENF/UFSM). gidias18@gmail.com
} 


\section{INTRODUÇÃO}

A enfermagem possui a responsabilidade direta pela assistência ao paciente durante 24 horas, constituindo-se na categoria profissional com o maior número de trabalhadores de saúde em estabelecimentos hospitalares (SILVA; LIMA; OLIVEIRA, 2016). A elaboração de um aporte legislativo, com destaque para a Lei do Exercício Profissional, foi fundamental para o avanço da profissão (CONSELHO..., 2008). Além disso, a prática de investigação científica, a qual delineia um corpo específico de conhecimento, configura-se como essencial para assegurar uma base de atuação sustentada e autônoma.

Nesta conjuntura, o enfermeiro assume diferentes atividades, sendo elas complexas, que envolvem o planejamento, a execução e a avaliação da assistência da enfermagem. Para tanto, necessita, inclusive, de competências gerenciais, ou seja, conhecimento científico, habilidade técnica, atitude e posicionamento, tendo em vista a prestação de uma assistência resolutiva e qualificada (ARBOIT; SILVA, 2013).

A atuação do enfermeiro ocorre em diversos locais dentro do ambiente hospitalar, incluindo Unidade de Terapia Intensiva Neonatal e Pediátrica. Em geral, as Unidades de Terapia Intensiva (UTI) têm uma rotina permeada por instabilidade, dúvidas e inseguranças (SILVA; LIMA; OLIVEIRA, 2016).

É um ambiente com inúmeros aparelhos, onde os pacientes se encontram em isolamento, na dependência de equipamentos e rotinas rígidas, em que são muito comuns situações de emergência e há iminente risco de morte (NASCIMENTO et al., 2017). É um ambiente rodeado de situações complexas, que demandam do enfermeiro atenção e dedicação, exigindo habilidades e conhecimento técnico-científico, disponibilidade física e emocional, ética e respeito, não apenas relacionado à criança, mas também à família e à equipe (BRAGA et al., 2015).

Por fim, a UTI é um ambiente diferente das outras unidades de internação, devendo, os enfermeiros que atuam nessas unidades, ser acessíveis, perceptíveis e preparados para atender às necessidades dos familiares (NASCIMENTO et al., 2017). Desta forma, vale ressaltar que a relação familiar/enfermagem é de suma importância, pois, durante o momento da hospitalização/internação, a enfermagem, ao realizar o acolhimento ao familiar, deve permitir que se estabeleça um diálogo bem como a criação de vínculo (NASCIMENTO et al., 2017).
A enfermagem ainda tem pouca visibilidade, pois a sociedade, em geral, desconhece o papel do enfermeiro e a importância de seus cuidados com o paciente (MARTINS; FERNANDES, 2014). Assim, a construção da visibilidade é um processo que exige dos profissionais engajamento e responsabilidade, tanto no aprimoramento das relações intergrupais quanto no sentido de busca do aprofundamento constante no campo do conhecimento teórico-prático (FROTA et al., 2015).

Vale ressaltar que a imagem do enfermeiro está ligada à trajetória histórica da profissão. Desta forma, os enfermeiros necessitam formar, planejar e sistematizar uma profissão com mais autonomia e decisão ante as áreas da política, social e institucional, de modo a ampliar a visibilidade da enfermagem (MARTINS; FERNANDES, 2014).

Diante do exposto, a realização de investigações que ofereçam suporte a discussões sobre a visibilidade do enfermeiro em unidade de cuidados intensivos neonatal e pediátrico são fundamentais, uma vez que os enfermeiros permanecem integralmente nestes setores, tendo grande parcela de responsabilidade no processo assistencial à saúde. Diante deste contexto, foi delineada a seguinte pergunta: Qual é a percepção de familiares de pacientes internados em Unidade de Terapia Intensiva neonatal e pediátrica sobre a visibilidade do enfermeiro? Nesse sentido, o estudo busca conhecer a percepção dos familiares sobre a visibilidade do enfermeiro atuante em Unidade de Terapia Intensiva neonatal e pediátrica.

\section{METODOLOGIA}

Trata-se de um estudo descritivo e exploratório de caráter qualitativo. A investigação foi realizada com familiares de pacientes que estiveram internados nas Unidades de Terapia Intensiva Pediátrica e Neonatal, em um hospital universitário de nível terciário localizado no interior do Estado do RS. A instituição é referência, na região, para o tratamento de média e alta complexidade.

Os participantes da investigação foram os familiares dos pacientes internados em Unidades de Terapia Intensiva Pediátrica e Neonatal. Foram incluídos os familiares dos pacientes que estivessem internados há mais de 72 horas no setor e em condições emocionais de verbalizar. Foram excluídos os familiares/acompanhantes que não tivessem vínculo de parentesco direto com o paciente. Todos os participantes foram convidados a participar voluntariamente da investigação 
e somente foram entrevistados após leitura e assinatura do Termo de Consentimento Livre e Esclarecido (TCLE).

Os dados foram produzidos por meio de entrevista semiestruturada com questões norteadoras acerca do tema investigado, às quais foram gravadas e transcritas. $O$ encerramento da produção dos dados se deu mediante o critério de saturação teórica.

Nesse contexto, os dados foram organizados e analisados com base no referencial proposto por Minayo (2014). A análise temática de conteúdo acontece em três etapas. A primeira etapa de pré-análise envolve a escolha dos documentos a serem analisados, bem como a leitura flutuante. A segunda consiste na exploração do material buscando-se alcançar a compreensão do texto, a fim de construir categorias temáticas. A terceira e última etapa diz respeito à interpretação dos resultados, de acordo com o referencial teórico-metodológico adotado, implicando na proposição de inferências e busca por significados, inter-relacionando-os com o quadro teórico-metodológico delineado (MINAYO, 2014).

O estudo seguiu todas as recomendações da Resolução 466\2012 do Conselho Nacional de Saúde (BRASIL, 2012), e foi aprovado pelo Comitê de Ética e Pesquisa (CEP) sob o número 335.012. Para garantir o sigilo e anonimato dos participantes da pesquisa, a identificação foi realizada pela letra E (entrevista) juntamente de números cardinais sequenciais, correspondente à ordem da entrevista (E1, E2, E3).

\section{RESULTADOS E DISCUSSÃO}

A pesquisa foi realizada por meio de entrevistas semiestruturadas com 12 familiares de pacientes internados nas Unidades de Terapia Intensiva neonatal e pediátrica, sendo seis entrevistados da UTI neonatal e seis entrevistados da UTI pediátrica. Dentre os participantes, 10 eram mães e 2 eram pais, todos com idade variando de 16 a 35 anos. Quanto à escolaridade, 25\% dos entrevistados tinham Ensino Fundamental completo, $8 \%$ Ensino Fundamental incompleto, 42\% Ensino Médio completo e $25 \%$ Ensino Médio incompleto.

Após a análise das entrevistas emergiu a seguinte categoria temática: "Visibilidade do trabalho do enfermeiro em Unidade de Terapia Intensiva neonatal e pediátrica".

Visibilidade do trabalho do enfermeiro em unidade de terapia intensiva neonatal e pediátrica

Nessa categoria, os participantes mencionaram suas percepções sobre a Unidade de Terapia Intensiva (UTI), bem como sobre o trabalho do enfermeiro ante as atividades de assistência prestada diretamente ao paciente. Além disso, destaca-se a visão de um profissional que desenvolve ações educativas e humanizadas, especialmente num contexto de gravidade.

A visibilidade profissional na (UTI) exige competência técnica e científica, congregando ao desenvolvimento de atividades assistenciais e gerenciais, cuja tomadas de decisão e adoção de condutas seguras estão diretamente relacionadas à vida e à morte das pessoas (FROTA et al., 2015).

Dessa forma, o processo de cuidar e gerenciar na área hospitalar pode ser considerado uma das principais grandezas do trabalho do enfermeiro. Neste contexto a UTI caracteriza-se por ser um cenário de inovação, onde a enfermagem presta atendimento a pacientes de alta complexidade, necessitando de profissionais com determinado perfil para atuar nessa área (SANTOS; CAMELO, 2015).

Os entrevistados, quando questionados sobre o seu entendimento e percepção em relação à UTI, mencionaram, em sua maioria, ser um lugar destinado à assistência 24 horas, local que cuida de pacientes muito graves e onde as crianças são bem-cuidadas.

"Eu entendo que uma UTI é onde as pessoas, crianças ou os adultos. precisam de mais cuidados, que tem mais aparelhagem do que em outros lugares" (E3).

"Para mim são cuidados mais profundos. Tem que ter os mínimos cuidados, tem que estar sempre observando, 24 horas do dia, não pode estar meio piscando, tem que estar meio de olho. Então é bem como diz: UTI, intensivo, 24 horas do dia tem que estar sempre de olho" (E10).

Neste estudo, os familiares souberam reconhecer a figura do enfermeiro atuante nas UTI - neonatal e pediátrica -, destacando a importância do papel do mesmo, reconhecendo as atividades que este desenvolve, bem como a sua habilidade em preparar o familiar para dar continuidade aos cuidados após a alta hospitalar, quando a criança retorna para casa. Este fato pode ser observado a seguir:

"Que eles deixam, que nem agora que ele [o paciente] está, deixam eu participar, ajudar, trocar fralda, dar de mamá" (E3).

"E curativo às vezes, tem curativos que são elas que fazem. Agora, estão me ensinando... presente [o enfermeiro] em quase todos os momentos, para tudo" (E7). 
Diante dessas considerações, percebe-se que o enfermeiro atuante nesta unidade presta atividade de ensino/orientações no manejo e cuidados específicos dos familiares com a criança. $O$ enfermeiro, quando fica próximo aos pais, prepara-os adequadamente para a continuidade do cuidado domiciliar, melhorando os indicadores de morbimortalidade infantil e a qualidade de vida dos neonatos e da família (SOARES et al., 2014).

Vale ressaltar que o enfermeiro atuante em UTI necessita sempre de uma qualificação adequada, ou seja, de uma busca constante do conhecimento científico, pois é ele quem realiza o planejamento do cuidado prestado aos pacientes. É ele quem orienta a família sobre o cuidado com o paciente. Este fato é relatado nas entrevistas:

"Como a minha filha está usando bolsinha [colostomia], me explicam como limpar, me mostram todas as coisas. O que eu não sei se seria a parte deles fazer isso... sempre quando vão trocar eles me chamam para ver como que é" (E2).

"...a enfermeira está sempre ali...agora estão me ensinando..., sempre a enfermeira está ali" (E7).

Desta forma, a atuação do enfermeiro na UTI neonatal é percebida, pelos familiares, como de extrema importância, na medida em que explica sobre os procedimentos, cuidados e esclarece dúvidas.

De acordo com os depoimentos, os familiares reconhecem a importância da presença e do trabalho do enfermeiro atuante nas UTI - neonatal e pediátrica. $O$ enfermeiro realiza orientações não somente no momento da internação, mas no convívio diário, pois ele acolhe o familiar desde o primeiro momento, executando o cuidado direto a estes pacientes nas 24 horas do dia, e expõem aos familiares toda logística de funcionalidade da unidade intensiva.

"... eu acho que eles teriam que ser mais reconhecidos. Porque eu digo, o médico é quem vai olhar, vai tipo dizer, dá a assinatura lá, mas quem faz tudo são os enfermeiros, eles que são quem cuidam exatamente, quem estão ali o dia inteiro em cima das crianças sempre" (E2).

"A enfermeira inexplicável, cansei de perguntar. Nenhuma nunca se negou, também dessa parte, excelente. 'Porque que isso, porque que não... Mãezinha é assim, assim, assim, assim'. Sabe? Elas têm paciência para te explicar. Eu acho que é o principal. Do profissionalismo delas de sentar e te explicar, tu pode ter diálogo com elas. Tu pode conversar, tu pode perguntar, tu pode tudo. Ali elas são maravilhosas para te explicar as coisas. Qualquer tipo de coisa" (E10).

Estes relatos permitem depreender que o profissional enfermeiro é percebido como uma presença constante no cuidado ao paciente e aos próprios familiares. Em um estudo realizado por Balbino et al. (2016), as mães relataram que a equipe de enfermagem ajuda a enfrentar a experiência de forma menos traumática, quando a recebe de maneira carinhosa e respeitosa, respondendo aos seus questionamentos, dúvidas e oferecendo explicações simples sobre determinados assuntos (BALBINO et al., 2016).

A visibilidade do enfermeiro é reconhecida pela sua articulação no processo assistencial, subsidiada pelo conhecimento científico e capacidade gerencial. Desta forma, o conhecimento científico, ligado ao fazer do enfermeiro, Ihe dá visibilidade, competência, confiança e respeito por parte da equipe (FROTA et al., 2015).

Os participantes apontam o enfermeiro como o profissional que tem maior conhecimento sobre os cuidados necessários à criança, na medida em que é visto como quem atua mais junto ao paciente, ficando a maior parte do tempo responsável pelos cuidados.

"... porque a gente vê que médico, ele vem, ele está sempre ali, mas quem cuida exatamente é $o$ enfermeiro. Ele que tem o contato maior com o paciente..." (E2).

"... cada criança tem uma enfermeira né? Uma enfermeira técnica no caso que cuida das crianças. E quando elas precisam de cuidados mais especiais alguma coisa, daí vem a enfermeira que é chefe... elas que tiram sangue, colocam as sondas nas criança..." (E3).

"...hãa é, a gente não faz nada bem dizer né, eu que gosto de tá trocando a minha filha né, de tá aquela coisa mais próxima, mas eles, a atividade deles ali é, é comparada que nem a nossa em casa, não desgrudam né, tão toda hora em cima..." (E11).

Desse modo, os familiares identificaram o enfermeiro como o profissional que realiza procedimentos específicos, que presta cuidados intensivos, que faz reuniões, fiscaliza e orienta sua equipe, bem como é um profissionais presente em todos os momentos.

"Suponhamos assim... na retirada de um cateter, curativo. Porque a maioria são os técnicos. Eles fazem, mas elas estão sempre em cima. 'Ah, você fez tal coisa? Ah, você lembra que tem que fazer isso'? Elas são bem-exigentes. A gente per- 
cebe que elas se preocupam. Elas têm amor pelo que fazem. Elas fazem reuniões, mas há bastante profissionalismo" (E10).

Nesta ótica, o enfermeiro é percebido como um profissional que se faz presente, estabelecendo um vínculo com o familiar desde o primeiro acolhimento ou contato, tornando-se um aliado no cuidado. A atuação em momentos de maior criticidade e em procedimentos mais complexos também foi destacada nos depoimentos:

\begin{abstract}
"Aqui eu vejo mais o contato das gurias da enfermagem com ele [o paciente]... porque assim, lá quem aspirava era a técnica, aqui geralmente... Às vezes é a enfermeira, às vezes é a técnica, mas ela está sempre mais presente. A enfermeira está sempre presente" (E7).

"... o enfermeiro na verdade eu acho que é nos casos onde está mais crítico algumas coisas porque quem dá banho é a técnica, elas dão banho, dão remedinho, dão mamá, e o enfermeiro mesmo, porque eu notei que a enfermeira que fica ali ela é mais chamada quando tem que pegar uma veia quando precisa botar uma sonda para quando precisa fazer uma coisa mais, mais específica" (E4).
\end{abstract}

Os entrevistados caracterizam o enfermeiro como o profissional que interage e identifica as situações de risco apresentadas pelo paciente, responsabilizando-se em direcionar informações acerca destas situações para os demais.

A atuação do enfermeiro e de sua equipe é de vital importância, pois estes estão preparados para identificar fatores de risco, bem como para instituir e praticar ações efetivas, essenciais e eficazes que tendem ao desenvolvimento do recém-nascido e à garantia de uma assistência humanizada e com qualidade (LIMA et al., 2013).

Majoritariamente, o cuidado humanizado de enfermagem está no olhar holístico ao paciente neonatal e pediátrico e sua família, que vai além de procedimentos técnicos, colocando-se disponível para a escuta qualificada e envolvimento do familiar nos cuidados (REIS et al., 2013). O cuidado humanizado, bem como a presença acolhedora do enfermeiro, foi evidenciado pelas falas.

"para mim, às vezes a gente recebe uma noticia que a gente não quer né, e eles notam, eles vêm, eles tentam te acolher de alguma forma, te dá uma palavra de conforto até. Uma enfermeira chegou para mim e disse assim: mãe tu que um ouvido? pra vê se aliviava um pouco a tensão daquele momento né, daí, mas eu achei super, achei muito importante, assim, achei muito querido da parte deles também" (E4).

Conforme Villa et al. (2017), na UTI a humanização tem um horizonte mais vasto, que engloba desde o ambiente físico até as relações entre os profissionais de saúde e pacientes/familiares (VILLA et al., 2017). Vale ressaltar que a Política Nacional de Humanização (PNH) foi lançada em razão das preocupações com questões pautadas ao atendimento à população nos serviços de saúde, sendo esta uma das prioridades nas políticas de saúde no Brasil (REIS et al., 2013).

Nesta perspectiva, a filosofia da humanização nas UTIs vem ganhando espaço, levando esse local a ser compreendido como o que permite a recuperação dos pacientes e não apenas como um ambiente destinado a pacientes sem chances de sobreviver (VILLA et al., 2017). Assim sendo, a enfermagem também ganha importância, pois esta conforta a família dos neonatos em cuidados intensivos e é responsável por um cuidado transpassado de carinho, afeto, dedicação, atenção e zelo, caracterizando um cuidado humanizado (SOARES et al., 2014). Em relação ao exposto, segue o depoimento:

"Quando eu cheguei... Eu olhava todo mundo de azul e digo: 'bah, qual será a chefe? Qual será a.... Assim, porque lá tinha essa diferença, né? A gente conversava mais era com as técnicas. E a chefe não, não ia muito, aí eu cheguei aqui e: 'bah, todo mundo de azul quem será?'. Aí depois quando eu fui conhecendo que eu fui ver como elas são todas companheiras, assim, não é porque elas são chefes. E nem as técnicas, que são só técnicas, são todo mundo companheiro. MINHAS (ênfase) companheiras, que eu chego e converso com qualquer uma, me entendo com qualquer um. Eu acho que é uma família aqui. Eu gosto muito, eu gostei muito daqui" (E7).

No depoimento, o familiar refere-se ao enfermeiro como sendo companheiro e tendo uma escuta qualificada, considerando a individualidade de cada um e importando-se com seus sentimentos. Ressalta-se que o processo de cuidado se torna humanizado quando são consideradas as peculiaridades e as especificidades de cada família.

Destaca-se que durante a internação é de suma importância a humanização da assistência à família e à criança. A equipe deve proporcionar cuidados integrais para ambos os membros, valorizando suas crenças e valores, lembrando que cada ser é único (LIMA et al., 2013). 
"Eu penso que nem todo o trabalhador; eles tão aqui trabalhando, só que é uma missão mais especial do que qualquer serviço porque tão cuidando de vidas, né. (pausa) Que nem no meu trabalho, eu trabalho com matéria-prima né, aqui eles trabalham com... com vidas" (E3).

Perante esse relato, percebe-se que ainda existe uma visão caritativa do enfermeiro pelo familiar, ou seja, a imagem do profissional está ligada a ser um profissional bondoso, paciente, religioso, remetendo que a profissão é um dom, "uma missão" e não uma formação profissional própria.

A imagem do enfermeiro reflete a própria construção da identidade, pois está associada a diversos preconceitos sem que exista uma imagem clara do que realmente é a profissão (MARTINS; FERNANDES, 2014). Desta forma, a visibilidade da enfermagem está relacionada à trajetória histórica da profissão, à falta de reconhecimento, ou seja, a construção da imagem da enfermagem é permeada por aspectos históricos, socioeconômicos e culturais (AVILA et al., 2013).

Vale ressaltar que a equipe de enfermagem que atua nas Unidades de Terapia Intensiva pediátrica e neonatal necessita ampliar suas capacidades e seus conhecimentos ante os cuidados, para assim ter uma assistência apropriada e com qualidade.

Por fim, a atuação do enfermeiro na UTI é de extrema importância, uma vez que este profissional possui formação, conhecimento e competência para planejar e prestar o cuidado bem como tomar decisões. Na percepção dos pais pesquisados, esta categoria revelou que os enfermeiros ganham importância, porque este é um profissional responsável por cuidar, acompanhar e prestar cuidados aos seus filhos.

\section{CONSIDERAÇÕES FINAIS}

A temática visibilidade do enfermeiro é de fundamental relevância, especialmente no que diz respeito à identidade social da profissão. Nesse sentido, o estudo buscou conhecer a percepção dos familiares diante da visibilidade do enfermeiro atuante em Unidade de Terapia Intensiva neonatal e pediátrica.

Os resultados permitiram concluir que as atividades desenvolvidas pelo enfermeiro em UTI pediátrica e neonatal são reconhecidas pelos entrevistados, especialmente as relacionadas à assistência direta ao paciente e às ações educativas; ainda, os participantes apontam os enfermeiros como profissionais de referência no que se refere ao cuidado e demais ações com a equipe. Destacaram, ainda, o fato de conside- rarem os enfermeiros profissionais confiáveis, dedicados, atenciosos, desenvolvendo cuidado humanizado aos pacientes.

Acredita-se na importância desta pesquisa, pois os resultados poderão favorecer a reflexão do enfermeiro acerca de sua prática, de modo a incentivar a busca de valorização profissional, uma vez que os enfermeiros permanecem integralmente nestes setores, tendo grande parcela de responsabilidade no processo assistencial à saúde.

\section{REFERÊNCIAS}

ARBOIT, É. L.; SILVA, L. A. A. Eventos adversos na enfermagem relacionados ao dimensionamento de pessoal. Revista de Enfermagem, n. 8, p. 128-139, 2013.

AVILA, L. I. et al. Implicações da visibilidade da enfermagem no exercício profissional. Rev. Gaúcha Enferm., v. 34, n. 3, p. 102-109, 2013.

BALBINO, F. S. et al. Percepção do cuidado centrado na família em unidade neonatal. Revista de Enfermagem da UFSM, v. 6, n. 1, p. 84-92, 2016.

BRAGA, L. C. et al. Demandas de atenção do enfermeiro em unidade de terapia intensiva pediátrica: uma investigação qualitativa. Arquivos de Ciências da Saúde, v. 22, n. 4, p. 5257, 2015.

BRASIL. Resolução 466/2012. Diretrizes e normas regulamentadoras de pesquisas envolvendo seres humanos. Brasília, DF: Ministério da Saúde, 13 de junho de 2012. Seção 1, p. 1-59.

CONSELHO FEDERAL DE ENFERMAGEM (Cofen). Ordem dos Enfermeiros. Regulamento do Exercício Profissional dos Enfermeiros. Decreto-lei no 104/98, de 21 de Abril de 1998. Disponível em: <https://www.ordemenfermeiros.pt/arquivo/legislacao/Documents/LegislacaoEnfermagem/REPE. pdf>. Acesso em: 21 dez. 2017.

FROTA, L. A. et al. A visibilidade do enfermeiro em unidades de terapia intensiva: percepções de trabalhadores. Revista Eletrônica de Enfermagem, v. 17, n. 3, 2015.

LIMA, A. C. et al. Sentimentos maternos frente à hospitalização de um recém-nascido na UTI Neonatal. Revista da Faculdade de Ciências Médicas de Sorocaba, v. 15, n. 4, p. 112-115, 2013.

MARTINS, M. J. R.; FERNANDES, S. J. D. A visibilidade da enfermagem, dando voz à profissão: revisão integrativa. Revista de Enfermagem Ufpe, on-line, v. 8, n. 7, p. 2.422-2.433, 2014. ISSN: 1981-8963.

MINAYO, M. C. S. O desafio do conhecimento: pesquisa qualitativa em saúde. 14. ed. São Paulo: Hucitec, 2014.

NASCIMENTO, E. R. P et al. As relações da enfermagem na unidade de terapia intensiva no olhar de Paterson e Zderad [Intensive care unit nursing relationships in the view of Paterson and Zderad]. Revista Enfermagem Uerj, v. 24, n. 2, p. 58-17, 2017. 


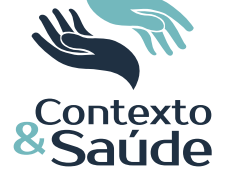

REIS, L. S et al. Percepção da equipe de enfermagem sobre humanização em unidade de tratamento intensivo neonatal e pediátrica. Revista Gaúcha de Enfermagem, v. 34, n. 2, p. 118-124, 2013.

SANTOS, F. C.; CAMELO, S. H. O enfermeiro que atua em Unidades de Terapia Intensiva: perfil e capacitação profissional. Cultura de los Cuidados, 3. ed., v. 19, n. 43, p. 127 140, 2015.

SANTOS, L. M. et al. Vivências maternas na unidade de terapia intensiva pediátrica. Revista de Pesquisa Cuidado é Fundamental, on-line, v. 5, n. 1, 2013.
Silviamar Camponogara - Camila Pinno - Constância Nenê Loiola Carla Silveira de Oliveira - Lenize Nunes Moura - Gisele Loise Dias

SILVA, A. E.; LIMA, P. K. M.; OLIVEIRA, C. Qualidade de vida dos profissionais de enfermagem nível médio em Unidade de Terapia Intensiva. Revista de Enfermagem do Centro-Oeste Mineiro, v. 6, n. 3, 2016.

SOARES, L. $\mathrm{G}$ et al. Enfermagem neonatal em cuidados intensivos: o olhar das famílias. Revista da Rede de Enfermagem do Nordeste, v. 15, n. 1, 2014.

VILLA, L. L. O et al. A percepção do acompanhante sobre o atendimento humanizado em unidade de terapia intensiva pediátrica. Rev. pesqui. cuid. fundam.(Online), v. 9, n. 1, p. 187-192, 2017. 